\title{
Better Understanding and Recognition of the Disconnects, Experiences, and Needs of Patients with Irritable Bowel Syndrome with Constipation (BURDEN IBS-C) Study: Results of an Online Questionnaire
}

Eamonn M. M. Quigley · John Horn • Michele Kissous-Hunt •

Robert A. Crozier · Lucinda A. Harris

Received: March 30, 2018 / Published online: June 26, 2018

(C) The Author(s) 2018

\section{ABSTRACT}

Introduction: The BURDEN IBS-C study was conducted to better understand the experiences, attitudes, and unmet needs of sufferers of irritable bowel syndrome with constipation (IBS-C) in comparison to the perceptions and challenges of healthcare providers (HCPs) who treat IBS-C patients.

Methods: This was an author-developed, online questionnaire using KnowledgePanel ${ }^{\circledR}$ to survey individuals with IBS-C $(N=1311)$. HCPs participated in a complementary online questionnaire and were recruited separately $(N=331)$. The study was fielded from June 29, 2016, to January 30, 2017.

Enhanced digital features To view enhanced digital features for this article go to https://doi.org/10.6084/ m9.figshare.6402053.

E. M. M. Quigley

Houston Methodist Hospital, Houston, TX, USA

J. Horn

University of Washington Medicine Pharmacy

Services, Seattle, WA, USA

M. Kissous-Hunt

Mount Sinai Gastroenterology, New York, NY, USA

R. A. Crozier

Synergy Pharmaceuticals Inc., New York, NY, USA

L. A. Harris $(\bowtie)$

Mayo Clinic Scottsdale, Scottsdale, AZ, USA

e-mail: Harris.Lucinda@mayo.edu
Results: Most patients had used (86\%) and/or were using $(76 \%)$ over-the-counter treatments for their IBS-C, with $12 \%$ currently on prescription therapy. At the time this study was conducted, $66 \%$ and $63 \%$ were not satisfied/completely satisfied with over-the-counter or prescription treatment, respectively, citing inadequate efficacy (55\%) and side effects (39\%), most commonly diarrhea, as common reasons for dissatisfaction. IBS-C respondents most commonly reported feeling frustrated (43\%) and stressed (28\%) regarding IBS-C, though $39 \%$ were accepting of IBS-C as part of daily life. HCPs were aligned with patients in thinking that patients were frustrated (76\%) and stressed (65\%) but HCPs were less likely to recognize that patients had become accepting of their IBS-C (13\%). Most HCPs (79\%) were not satisfied/completely satisfied with the prescription treatments available at the time this study was conducted. Inadequate response rates to current therapies (55\%) and treatment adherence/compliance issues (58\%) were the most frequent challenges encountered by HCPs. IBS$\mathrm{C}$ respondents reported that their symptoms impacted productivity and personal activity, on average, 4 and 3 days/month, respectively.

Conclusion: These results suggest that current management pathways may not be adequately addressing the symptoms and needs of individuals with IBS-C, most notably side effects and lack of efficacy. Patients and HCPs expressed dissatisfaction with over-the-counter and 
prescription treatments available at the time this study was conducted. Additional treatment options and improved dialogue would be beneficial to HCPs and patients.

Funding: Synergy Pharmaceuticals Inc.

Keywords: Gastroenterology; Irritable bowel syndrome with constipation; Quality of life; Survey

\section{INTRODUCTION}

Irritable bowel syndrome with constipation (IBS-C) is a chronic gastrointestinal disorder affecting approximately $5 \%$ of the US population $(\sim 16$ million people), though prevalence may be underestimated as many people exhibit IBS-C symptoms without seeking medical care and thus never receive a formal diagnosis $[1,2]$. Trinkley et al. identified a total of 28.2 million office visits for IBS-C occurring between 2000 and 2009 [3], resulting in a significant healthcare burden. According to Rome IV criteria, IBS$\mathrm{C}$ is defined by recurrent abdominal pain associated with defecation and onset associated with reduced stool frequency and/or lumpy/ hard stools [2]. For clinical practice, the American College of Gastroenterology states that IBS$\mathrm{C}$ is characterized as abdominal discomfort associated with altered bowel habits [4].

There is limited data on the evolution and impact, from the sufferer's perspective, of IBS-C in the United States, especially comparing patient experiences to the perceptions of healthcare providers (HCPs). The current study, BURDEN IBS-C (Better Understanding and Recognition of the Disconnects, Experiences, and Needs of Patients with Irritable Bowel Syndrome with Constipation), is the companion of the BURDEN-CIC study that explored these issues among subjects with chronic idiopathic constipation (CIC) and that was recently published [5]. The BURDEN IBS-C study was designed to develop a better understanding of the experiences, attitudes, and unmet needs of individuals with IBS-C and to assess the understandings of the HCPs who treat these patients. How the findings of BURDEN IBS-C relate to those of the BURDEN-CIC study will be discussed.

\section{METHODS}

The methodology for the BURDEN IBS-C study was nearly identical to that of the BURDEN-CIC study [5], with the only difference being the population disease state (i.e., IBS-C or CIC) studied. The BURDEN IBS-C study utilized 2 author-developed online questionnaires-one for adults with IBS-C and another for HCPs who treat patients with IBS-C-in order to evaluate the impact of IBS-C on quality of life (QOL) and daily activities, as well as treatment experience and satisfaction, in a representative sample of the US population. The patient protocol and associated materials were reviewed and approved by the Western Institutional Review Board. GfK conducted both the patient and HCP questionnaires in accordance with globally accepted standards of good clinical practice (as defined in the International Conference on Harmonisation E6 Guideline for Good Clinical Practice), in accordance with the Health Insurance Portability and Accountability Act, and in keeping with local regulations. Informed consent was obtained from all respondents before being included in the study. Panelists who met the inclusion criteria and none of the exclusion criteria completed an online, self-administered questionnaire that was estimated to take $45 \mathrm{~min}$.

\section{Patient Questionnaire}

The BURDEN IBS-C patient questionnaire was author-developed and IRB-approved, with panelists recruited and surveyed from June 29, 2016, to January 30, 2017. To identify adults suffering with IBS-C, the BURDEN IBS-C study used a proprietary consumer panel (KnowledgePane ${ }^{\circledR}$ ) in conjunction with an additional opt-in panel. KnowledgePanel ${ }^{\circledR}$ is a representative sample of the total US population, accounting for demographic and epidemiological factors, including age, race, marital status, education, employment status, and geographic region. Participants were required to be able to 
read and understand an informed consent document and were then given the option to continue with the online questionnaire. All responses were confidential, with any identifying information removed. Panelists were told only that they were answering a questionnaire about their health when initially being screened for participation and that they had the option of not answering specific questions and could leave the survey at any time.

Panelists eligible to participate in the patient questionnaire were aged $\geq 18$ years. Regarding diagnosis, panelists either reported that they had been told by an HCP that they were formally diagnosed with IBS-C (i.e., the diagnosed group) or fulfilled Rome IV criteria for IBS-C as determined in the screening section of the questionnaire (i.e., the undiagnosed group). Respondents were not eligible to participate in BURDEN IBS-C if they had CIC, irritable bowel syndrome with diarrhea or mixed diarrhea and constipation, inflammatory bowel disease, diverticulitis, diverticulosis, celiac disease, or cancer of the gastrointestinal tract, or if they had regularly taken an opioid (narcotic) within the past 3 months.

Enrolled panelists completed an online, selfadministered questionnaire, with answer formats that included dichotomous, multiplechoice, and open-ended options, as well as numeric rating scales (1-5 or 1-7). As part of the survey, participants were asked to report the symptomatology of their IBS-C, the impact of IBS-C symptoms on activities of daily living and QOL, their experience with HCP interactions, and their experience with IBS-C treatments, including satisfaction.

Data were internally validated to ensure that respondents completed the questionnaire only once, that sufficient time was spent completing the questionnaire, and that there was a consistent response pattern. Any incomplete study data were removed from the overall database. Demographic and geographic distributions from the Current Population Survey were used as benchmarks [6].

\section{HCP Questionnaire}

HCPs were recruited independently of participants in the patient questionnaire, with the survey fielded from July 20 to August 10, 2016. The HCP questionnaire, which was based on the patient questionnaire, was targeted to a random national sample of gastroenterologists (GEs), primary care physicians (PCPs), nurse practitioners (NPs), and physician assistants (PAs) who actively treat patients with IBS-C. Eligible HCPs included those who had been practicing medicine for $2-40$ years, spent at least $75 \%$ of their time in direct patient care, saw at least 50 patients in total in a typical month (including $\geq 15$ patients with IBS-C), had prescription privileges (if an NP or PA), and had prescribed at least 10 medications for IBS-C in the month prior to the survey. HCPs were provided with a modest honorarium for participation. Question and answer formats were the same as those used in the patient questionnaire.

\section{RESULTS}

\section{Respondents}

Of the 29,665 clicks on the patient questionnaire link, 27,709 panelists completed the screening test, with 1311 panelists qualifying for and completing the survey. Most respondents were female $(73 \%)$, were Caucasian (nonHispanic; 65\%), and had at least a high school education (95\%) (Table 1). The mean age of respondents was 46 years, with a mean age of symptom onset of 44 years. In addition, the majority of respondents $(71 \%)$ reported that they had been diagnosed by an HCP, with the remainder (29\%) not receiving a formal diagnosis but rather fitting the Rome IV criteria for IBS-C based on responses to the screening questionnaire (undiagnosed respondents).

The HCP questionnaire was completed by 331 HCPs (155 GEs, 76 PCPs, 50 NPs, and 50 PAs). The majority of HCPs were male (63\%), had been in clinical practice for an average of 17.5 years, and spent an average of $96.8 \%$ of their time in direct patient care (Table 2). 
Table 1 IBS-C respondent demographics

Total respondents $N=1311$

Female $73 \%$

Age, years, mean $(\mathrm{SD})$

$46.0(15.6)$

Age at symptom onset, years, mean (SD)

$43.6(16.0)$

Diagnosed by an HCP

Race/ethnicity

White/non-hispanic

Black/non-hispanic

$10 \%$

Mixed/non-hispanic $5 \%$

Other/non-hispanic $4 \%$

Hispanic $17 \%$

Highest education level

High school—no graduation

High school-diploma/GED $31 \%$

College-no graduation $23 \%$

College-graduation $30 \%$

College-post-graduate

Current treatment to manage IBS-C symptoms

Lifestyle changes

General diet changes/home remedies $28 \%$

Increased activity and exercise $21 \%$

Meditation/yoga/relaxation techniques $8 \%$

Gluten-free diet $7 \%$

OTC treatment remedies

Fiber $27 \%$

Stool softeners $13 \%$

Probiotics/prebiotics $22 \%$

Non-prescription laxatives

Non-prescription stimulant laxatives

Other

Prescription therapy

$12 \%$

$C I C$ chronic idiopathic constipation, GED General Educational Development, $H C P$ healthcare provider, $I B S-C$ irritable bowel syndrome with constipation, $O T C$ over the counter, $S D$ standard deviation 
Table 2 Healthcare provider demographics

\begin{tabular}{|c|c|c|c|c|}
\hline & $\begin{array}{l}\text { GEs } \\
N=155\end{array}$ & $\begin{array}{l}\text { PCPs } \\
N=76\end{array}$ & $\begin{array}{l}\text { NPs } \\
N=\mathbf{5 0}\end{array}$ & $\begin{array}{l}\text { PAs } \\
N=\mathbf{5 0}\end{array}$ \\
\hline Males & $84 \%$ & $75 \%$ & $8 \%$ & $34 \%$ \\
\hline Age, years, mean $(\mathrm{SD})$ & $48.8(8.2)$ & $50.7(5.9)$ & $48.6(7.6)$ & $44.3(9.0)$ \\
\hline Years in clinical practice, mean (SD) & $17.3(7.4)$ & $20.2(6.1)$ & $15.0(5.5)$ & $16.2(7.2)$ \\
\hline Time spent in direct patient care, mean (SD) & $96.5 \%(5.1 \%)$ & $97.6 \%(4.2 \%)$ & $97.0 \%(5.6 \%)$ & $96.4 \%(5.6 \%)$ \\
\hline \multicolumn{5}{|l|}{ Practice setting } \\
\hline Community practice & $62 \%$ & $57 \%$ & $34 \%$ & $40 \%$ \\
\hline Solo practice & $21 \%$ & $32 \%$ & $30 \%$ & $48 \%$ \\
\hline Hospital-based practice & $9 \%$ & $8 \%$ & $18 \%$ & $10 \%$ \\
\hline Academic practice & $8 \%$ & $3 \%$ & $8 \%$ & - \\
\hline \multicolumn{5}{|l|}{ Medical specialty } \\
\hline Primary care/general practice & - & $100 \%$ & $38 \%$ & $34 \%$ \\
\hline Gastroenterology & $100 \%$ & - & $38 \%$ & $53 \%$ \\
\hline Internal medicine & - & - & $18 \%$ & $12 \%$ \\
\hline
\end{tabular}

$G E$ gastroenterologist, $N P$ nurse practitioner, $P A$ physician assistant, $P C P$ primary care physician, $S D$ standard deviation

Table 3 How IBS-C respondents feel about their IBS-C and HCP perception of patients' attitudes

\begin{tabular}{lllll}
\hline & \multicolumn{2}{l}{ IBS-C respondents } & & \\
\cline { 2 - 4 } & Diagnosed (\%) & Undiagnosed (\%) & Total (\%) & HCPs (\%) \\
\hline Frustrated & 44 & 40 & 43 & 76 \\
Accepting as part of daily life & 41 & 36 & 39 & 13 \\
Stressed & 31 & 23 & 28 & 65 \\
Self-conscious & 23 & 20 & 22 & 33 \\
Fed up & 23 & 16 & 21 & 47 \\
Embarrassed & 22 & 21 & 21 & 40 \\
In control & 22 & 18 & 20 & 6 \\
Fine, no big deal & 12 & 22 & 16 & 5 \\
Obsessed with symptoms & 9 & 7 & 8 & 48 \\
\hline
\end{tabular}

$H C P$ healthcare provider, $I B S-C$ irritable bowel syndrome with constipation

\section{Burden of IBS-C}

IBS-C respondents primarily reported negative emotive terms when describing how they felt about their IBS-C and its symptoms (Table 3). Most commonly, IBS-C respondents reported feeling frustrated and stressed regarding their IBS-C; however, nearly $40 \%$ of respondents 


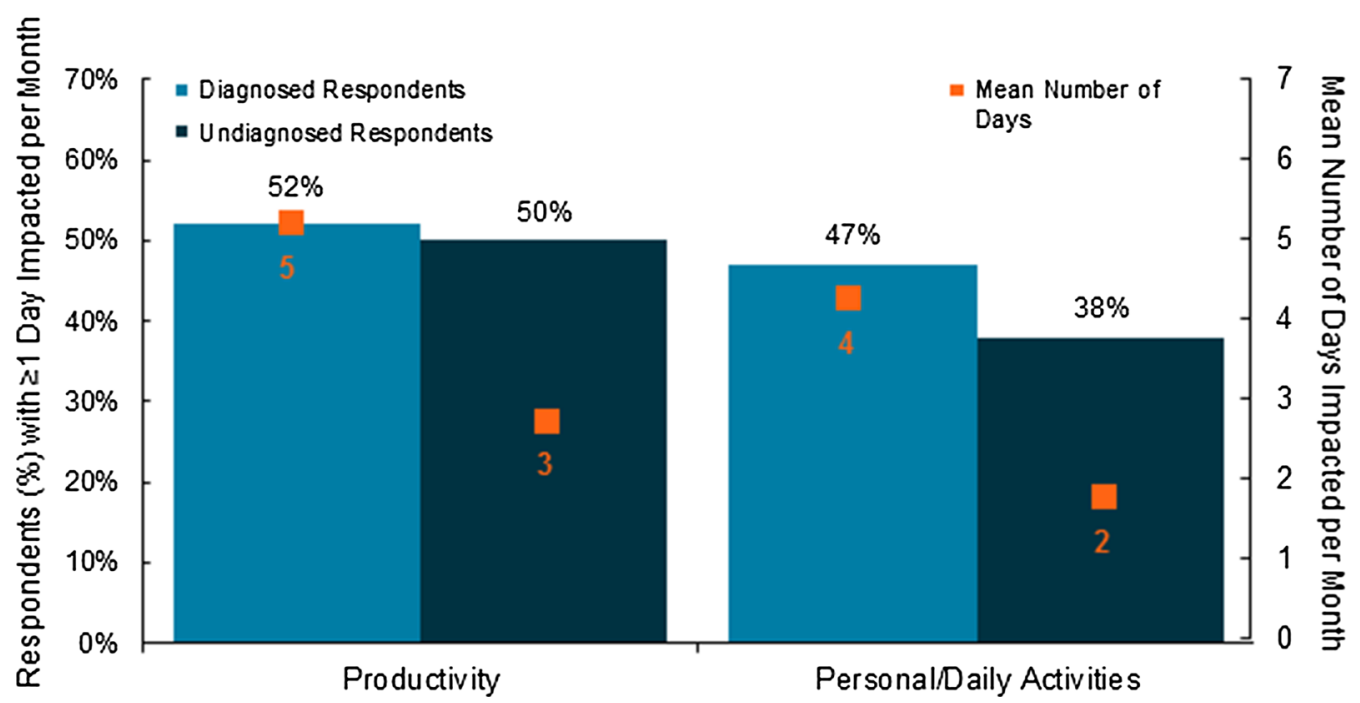

Fig. 1 IBS-C association with impacted productivity and personal activities. Mean number of days impacted includes respondents whose estimate was 0

reported that they were accepting of IBS-C as part of their daily life. This trend was similar between the diagnosed and undiagnosed groups, with the exceptions that more undiagnosed respondents reported feeling fine, no big deal and more diagnosed patients reported feeling stressed. HCPs agreed that patients were frustrated and stressed, but HCPs were less likely to recognize that patients had become accepting of their IBS-C and were more likely to believe patients were obsessed with symptoms. Although $20 \%$ of the IBS-C respondents thought they were in control of their IBS-C, only $6 \%$ of HCPs thought their typical IBS-C patient was in control of their symptoms. Of the total IBS-C respondent population, 59\% described their symptoms as somewhat to extremely bothersome. As expected, diagnosed patients more than undiagnosed respondents reported their symptoms as somewhat to extremely bothersome (64\% and 52\%, respectively).

About half of respondents reported that their productivity (work/school) and/or personal activity (social events/hobbies) was impacted by IBS-C symptoms for at least 1 day in a typical month (Fig. 1). Diagnosed patients reported that their productivity and personal activity was impacted 5 and 4 days/month, respectively. These values increased to 8 and 7 days, respectively, in those who reported at least
1 day/month impacted. Undiagnosed respondents reported that their productivity and personal activity was impacted 3 and 2 days/month, respectively. These values increased to 5 and 4 days/month, respectively, in those who reported at least 1 day/month impacted. HCPs estimated averages of 9 days/month and 4 days/month for productivity and personal activity impairment, respectively. In all, 13\% of respondents reported that their IBS-C symptoms caused them to miss at least 1 day of work or school in a typical month. In addition, $13 \%$ of respondents indicated they had been to the emergency department at least once in the past year for their constipation symptoms, with $20 \%$ of diagnosed and $6 \%$ undiagnosed respondents indicating at least 1 visit. HCPs reported that a similar percentage of their patients visited the emergency department for their IBS-C symptoms, with similar rates noted across HCP type (range 16-21\%), which aligned with what patients reported.

\section{IBS-C Management Pathway}

Respondents reported experiencing multiple stool and abdominal symptoms at the onset of their IBS-C (Table 4), with diagnosed patients 
Table 4 Symptoms first experienced by respondents suffering with IBS-C

\begin{tabular}{|c|c|c|c|}
\hline & $\begin{array}{l}\text { Diagnosed } \\
(\%)\end{array}$ & $\begin{array}{l}\text { Undiagnosed } \\
(\%)\end{array}$ & $\begin{array}{l}\text { Total } \\
(\%)\end{array}$ \\
\hline \multicolumn{4}{|c|}{ Abdominal symptoms } \\
\hline $\begin{array}{l}\text { Abdominal } \\
\text { discomfort }\end{array}$ & 66 & 51 & 60 \\
\hline $\begin{array}{l}\text { Abdominal } \\
\text { bloating/ } \\
\text { distension }\end{array}$ & 65 & 48 & 58 \\
\hline Abdominal pain & 62 & 52 & 58 \\
\hline Stomach cramps & 54 & 41 & 48 \\
\hline Nausea & 25 & 11 & 19 \\
\hline \multicolumn{4}{|l|}{ Stool symptoms } \\
\hline $\begin{array}{l}\text { Difficulty with } \\
\text { bowel } \\
\text { movement }\end{array}$ & 75 & 67 & 72 \\
\hline Straining & 58 & 47 & 54 \\
\hline $\begin{array}{l}\text { Hard, lumpy, or } \\
\text { pebble-like } \\
\text { stools }\end{array}$ & 53 & 46 & 50 \\
\hline $\begin{array}{l}\text { Feeling of } \\
\text { incomplete } \\
\text { bowel } \\
\text { movement }\end{array}$ & 53 & 43 & 49 \\
\hline Infrequent stools & 41 & 30 & 37 \\
\hline
\end{tabular}

IBS-C irritable bowel syndrome with constipation

reporting being more symptomatic than undiagnosed respondents (mean of 6.0 symptoms vs 4.6 symptoms, respectively). As expected, the majority of patients first experienced abdominal symptoms (including discomfort, bloating/distension, and pain), with most patients also experiencing difficulty with bowel movements. The largest differences, favoring diagnosed over undiagnosed respondents, were for the symptoms of abdominal bloating/distension (difference, $17 \%$ ) and abdominal discomfort (difference, 15\%).
Table 5 Symptom management prior to consulting an HCP and initial HCP recommendations for the treatment of constipation symptoms

IBS-C respondents ${ }^{a}$

\begin{tabular}{ll}
\hline Prior to & Initial HCP \\
HCP (\%) & recommendation
\end{tabular}

(\%)

Dietary changes/home remedies

$\begin{array}{lll}\text { Gluten-free diet } & 6 & 8 \\ \text { FODMAP diet } & 0 & 2 \\ \text { Other }^{\text {b }} & 33 & 50 \\ \text { Increased exercise } & 20 & 39 \\ \text { meditation/yoga } & 4 & 8 \\ \text { Probiotics or prebiotics } & 17 & 24 \\ \text { Fiber } & 39 & - \\ \text { Stool softeners } & 24 & -\end{array}$

Non-prescription laxative treatment

$\begin{array}{lll}\text { Osmotic laxative } & 16 & - \\ \text { Stimulant laxative } & 14 & - \\ \text { Continue current OTC } & - & 17 \\ \text { medicine } & & \\ \begin{array}{l}\text { Continue current OTC } \\ \text { in a different manner }\end{array} & & 10 \\ \text { Different OTC laxative } & - & 14 \\ \text { Prescription IBS-C } & - & 22 \\ \text { treatment } & \end{array}$

No recommendation $\quad-\quad 4$

Respondents answered both questions using a multiplechoice drop-down menu answer format

FODMAP fermentable oligo-, di-, monosaccharides and polyols, $H C P$ healthcare provider, IBS-C irritable bowel syndrome with constipation, $O T C$ over the counter

${ }^{a}$ Of respondents who have discussed symptoms with an HCP and who were HCP-diagnosed with IBS-C

b Includes general diet changes, caffeine, prunes, milk of magnesia, laxative teas, mineral oil, etc

${ }^{c}$ Different manner refers to a change in frequency or dose amount 
All diagnosed patients had consulted an HCP regarding their symptoms; however, $61 \%$ of undiagnosed respondents had never discussed their symptoms with an HCP, including pharmacists. For those who consulted an HCP, the symptoms most commonly reported to have led to a discussion with an HCP were abdominal pain/discomfort $(68 \%)$, difficulty with bowel movements (44\%), and abdominal bloating/ distension (34\%). More diagnosed respondents than undiagnosed respondents indicated difficulty with bowel movements as one of the trigger symptoms ( $46 \%$ vs $33 \%$, respectively). Of respondents indicating that two or more symptoms led to an HCP discussion, the trigger symptom was most commonly abdominal pain (28\%).

Prior to reaching out to their HCP, IBS-C sufferers mostly tried fiber, general dietary changes/home remedies, stool softeners, and increased exercise (Table 5). Of note, general dietary changes/home remedies, and increased exercise were also the recommendations that respondents indicated their HCP made during the first discussion for treatment considerations (Table 5). In addition, about a quarter of the diagnosed respondents initially received a recommendation of probiotics/prebiotics (24\%) and/or prescription treatment $(22 \%)$ by their HCP.

In terms of current treatment, non-prescription IBS-C treatments were being used by $76 \%$ of respondents (diagnosed, 79\%; undiagnosed, $71 \%$ ), with only $12 \%$ of respondents currently taking a prescription treatment for their IBS-C symptoms (not mutually exclusive groups), despite $22 \%$ of respondents reporting an initial recommendation for a prescription treatment. Interestingly, $21 \%$ of respondents with IBS-C (diagnosed, 18\%; undiagnosed, 26\%) reported that they were not currently using any treatment for their constipation symptoms. Of those

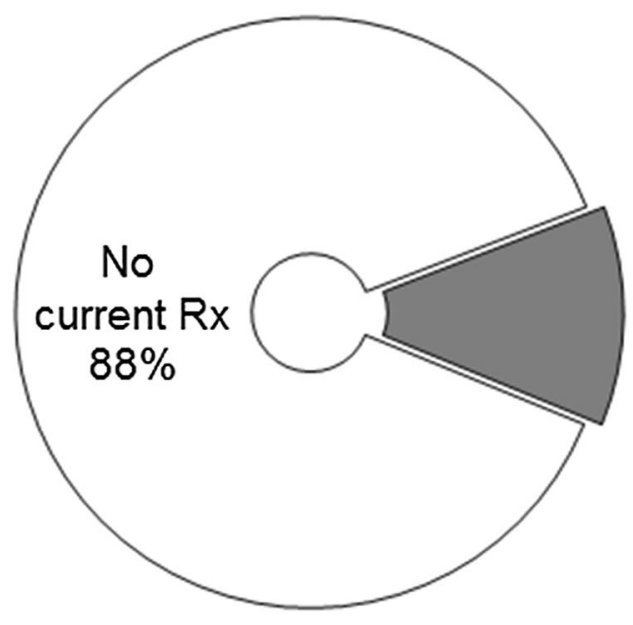

\section{Patients ever using branded Rx treatment}

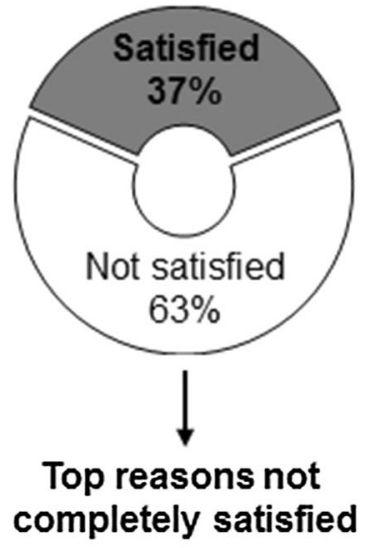
Current $\mathrm{Rx}$ $12 \%$

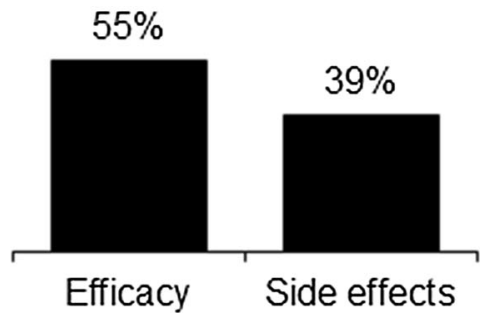

Fig. 2 Patient experience with prescription therapies for the treatment of IBS-C. Left: Of respondents who were past or current users of prescription treatment. Right: Of

patients who were past or current users of branded prescription, which includes lubiprostone and linaclotide. $R x$ prescription 
Table 6 Residual IBS-C symptoms despite prescription treatment

\begin{tabular}{ll}
\hline & $\begin{array}{l}\text { IBS-C respondents } \\
(\%)\end{array}$ \\
\hline Abdominal symptoms & \\
Bloating/distension & 64 \\
Abdominal discomfort & 48 \\
Abdominal pain & 28 \\
Nausea & 30 \\
Stomach cramps & 23 \\
Stool symptoms & \\
Hard, lumpy, or pebble-like & 32 \\
stools & \\
Feeling of incomplete BM & 33 \\
Difficulty with BM & 23 \\
Straining & 22 \\
Infrequent stools & 18 \\
\hline
\end{tabular}

$I B S$ - $C$ irritable bowel syndrome with constipation

${ }^{a}$ Patients who were taking a prescription treatment at the time of the questionnaire and indicated that their constipation symptoms were not relieved all the time with that prescription

using over-the-counter (OTC) treatments for their IBS-C, only about one-third (36\%) was satisfied or completely satisfied with treatment (diagnosed, 37\%; undiagnosed, 32\%). When describing their experience with OTC treatments, respondents primarily indicated that: their bowel movements were unpredictable (39\%), at least 1 day/week was dedicated to using their OTC laxative (33\%), and the laxative caused gassiness $(26 \%)$ or diarrhea (22\%).

\section{Patient Experience with Prescription IBS-C Treatments}

Of past and current users of branded prescription IBS-C treatment available at the time the study was conducted (linaclotide or lubiprostone), $37 \%$ reported being satisfied or completely satisfied, resulting in $63 \%$ of IBS-C patients potentially seeking new treatment options (Fig. 2). The primary reasons for dissatisfaction were issues with efficacy (55\%) and side effects (39\%), with $23 \%$ of patients specifically identifying diarrhea as the reason for their dissatisfaction.

Despite taking a prescription IBS-C treatment, $77 \%$ of patients still experienced residual abdominal- and stool-related symptoms (Table 6). Of these, abdominal bloating/distension was the most frequent residual symptom, followed by abdominal discomfort, suggesting that prescription treatments available at the time of this study may be effective at reducing abdominal pain, but may be less effective in reducing other common abdominal symptoms. Respondents also reported residual stool symptoms, such as hard, lumpy, or pebble-like stools and feeling of incomplete bowel movements.

\section{Challenges in Managing IBS-C}

HCPs experience many challenges with managing IBS-C symptoms that varied according to HCP type (Table 7). They reported inadequate efficacy (55\%) and patient adherence/compliance (58\%) as the most common challenges, with NPs and PAs reporting the highest levels of patient adherence/compliance challenges. Only $21 \%$ of HCPs were satisfied or completely satisfied with prescription IBS-C treatments available at the time of this study, citing, in addition to inadequate efficacy reported above, the management of treatmentrelated diarrhea (41\%) as a challenge most frequently experienced in treating IBS-C. Of the HCPs, $89 \%$ did not agree with the statement that diarrhea is an acceptable outcome of IBS-C treatment. Only $16 \%$ of HCPs agreed that diarrhea is a sign that the IBS-C treatment is working, comprising $25,13,8$, and $4 \%$ of GEs, PCPs, NPs, and PAs, respectively, with $77 \%$ of patients who experienced residual diarrhea not agreeing with the statement that diarrhea is an acceptable outcome of taking a medication to relieve symptoms of constipation. Only $22 \%$ of patients agreed that diarrhea is a sign that their IBS-C medication(s) is/are working. 
Table 7 Greatest perceived challenges by healthcare providers in the management of patients with IBS-C

\begin{tabular}{llllll}
\hline & GEs (\%) & PCPs (\%) & NPs (\%) & PAs (\%) & Total (\%) \\
\hline Inadequate treatment response & 66 & 53 & 46 & 32 & 55 \\
Patient adherence/compliance & 55 & 49 & 70 & 70 & 58 \\
Lack of IBS-C-specific treatment options & 45 & 45 & 30 & 30 & 40 \\
IBS-C treatment-related diarrhea & 41 & 33 & 50 & 42 & 41 \\
Other IBS-C treatment-related side effects & 33 & 28 & 30 & 36 & 32 \\
Lack of clarity on criteria for adequate treatment response & 26 & 38 & 36 & 24 & 30 \\
Lack of IBS-C treatment guidelines & 18 & 33 & 32 & 14 & 23 \\
\hline
\end{tabular}

$G E$ gastroenterologist, $I B S-C$ irritable bowel syndrome with constipation, $N P$ nurse practitioner, $P A$ physician assistant, $P C P$ primary care physician

HCPs estimated that $38 \%$ of their patients using prescription IBS-C treatments take treatment holidays (i.e., stop taking their prescription treatment for a period of time) without being instructed to do so, with similar estimates across HCP type. Of IBS-C patients taking a prescription treatment, $67 \%$ indicated having stopped taking their treatment at least once without HCP instruction, with the most common reason being that they forgot to take the medication (46\%). Other common reasons included "I could not take the risk of having diarrhea" (30\%) and "I usually plan taking my medication around when I want/need to have bowel movement" (30\%). The most common reasons indicated by HCPs for patients taking a prescription treatment holiday included: patients want to try managing their symptoms without medication (64\%), patients cannot afford medication (55\%), patients experience side effects (53\%), patients feel they no longer need a medication when their symptoms of constipation appear to be under control (53\%), patients forget to take medication (50\%), and patients don't want to take a risk of having diarrhea (46\%).

\section{DISCUSSION}

The BURDEN IBS-C study confirmed the physical, psychosocial, and quality of life impact of
IBS-C revealed in other surveys [7-9], emphasizing the substantial burden associated with IBS-C, regardless of treatment. Those with IBS-C experience many bothersome symptoms that significantly and directly impacted QOL, productivity, and personal activity. In general, IBS$C$ respondents were not satisfied with the OTC and prescription treatments evaluated in this study, citing poor efficacy and diarrhea as the primary reasons for dissatisfaction. Fewer than half of the patients who initially received the recommendation of a prescription therapy by their HCP were currently taking a prescription treatment, suggesting a barrier with taking and/ or obtaining prescription treatment. Of the $12 \%$ who were currently using a prescription IBS-C treatment for symptoms, the majority (77\%) still experienced IBS-C symptoms despite treatment. In addition, HCPs reported low levels of satisfaction with prescription treatments, which may affect their willingness to prescribe them.

Patients were frustrated and stressed regarding their IBS-C, which was well recognized by HCPs. However, a large proportion of patients reported that they were accepting of their IBS-C, which was not well recognized by HCPs. This attitudinal disconnect may be a problem for patients in achieving optimal care and can result in patients searching for an HCP who better understands their needs. This difference in perception was also noted between HCPs and patients with CIC in the BURDEN-CIC study [5]. 
One-fifth $(21 \%)$ of respondents in the BURDEN IBS-C study were not using any treatment for their IBS-C symptoms, supporting the observed frustrated-yet-accepting attitude toward IBS-C. Despite a variety of pharmacological approaches to treatment, only $20 \%$ of respondents felt they were in control of their IBS-C symptoms and even fewer HCPs (6\%) felt their patients were in control. The perceived lack of control observed in both BURDEN-CIC and BURDEN IBS-C was reflected by the substantial number of days with work/school and personal activity negatively impacted as reported by the respondents.

Approximately one-fourth (29\%) of the IBS$\mathrm{C}$ respondent population was classified as having IBS-C symptoms using the Rome IV criteria [2]. Generally, undiagnosed and diagnosed IBS$\mathrm{C}$ respondents appeared to be similar in how they felt about their IBS-C, with the exception that more undiagnosed respondents reported feeling fine, no big deal-a difference in attitude that may partly explain why the undiagnosed respondents remain undiagnosed. Expectedly, fewer undiagnosed patients indicated that their symptoms were bothersome, and undiagnosed patients reported fewer days on average with their productivity and/or personal activity impaired due to symptoms. Although gender differences were not evaluated in this study, other studies have examined this issue. The prevalence of IBS-C tends to be higher in women than in men $(2: 1$ ratio) $[1,10]$, and women tend to report more severe symptoms than men, especially abdominal symptoms [11-13]. Women and men may also be impacted differently in their daily life by symptoms [14], though gender may not be a significant predictor of health-related quality of life [15] or patient satisfaction [16].

For both BURDEN populations, HCPs tended to initially recommend lifestyle changes and OTC treatments, typically with some modifications to what patients may have already tried prior to the consultation. HCPs were less likely to initially recommend that an IBS-C patient continue their OTC therapy (at the same dose/ frequency or at a different dose/frequency) than their CIC patients and were more likely to recommend probiotics/prebiotics to IBS-C patients. However, rates of all other initial recommendations were similar between the two populations. Though a similar proportion of IBS-C and CIC respondents indicated satisfaction with prescription treatment, IBS-C patients more frequently experienced residual symptoms compared with CIC patients, especially abdominal symptoms (e.g., pain, discomfort, and bloating). More than twice as many IBS-C patients reported residual abdominal bloating/ distension than did CIC patients. In addition, more IBS-C patients reported experiencing residual hard, lumpy, or pebble-like stools than did CIC patients (34\% vs $21 \%$ ), yet fewer IBS-C patients experienced difficulty with bowel movements (23\% vs 35\%) [5]. Abdominal bloating was associated with worse quality of life, treatment satisfaction, and treatment responsiveness [17]; therefore, the finding that more than half of patients still experienced abdominal bloating/distension despite current treatment emphasizes the importance and difficulty of addressing all of the symptoms in this heterogeneous disorder.

Both IBS-C and CIC sufferers reported similar assessments of their feelings toward their condition, its negative impact on productivity and personal activities, and their dissatisfaction with available treatments. IBS-C respondents tended to report more abdominal symptoms when describing the onset of disease, while CIC respondents more frequently reported stool symptoms [5], supporting the concept that these disorders exist on a continuum of functional bowel disorders, primarily defined by the presence or absence of abdominal pain [2]. Though IBS-C is a heterogeneous disorder that can overlap with CIC, respondents were aligned in their general dissatisfaction of currently available treatments, and most HCPs also felt that more effective and tolerable treatments are needed.

Although the BURDEN IBS-C study enrolled a large number of IBS-C and HCP respondents, the results may have limitations. As with all online surveys, patient selection may be limited to those with interest in and access to such a survey. However, GfK, the company that administered the survey, uses statistical methods to align the survey participants with US 
Current Population Survey benchmarks and will provide a computer and Internet access free of charge to those who do not have Internet access. In addition, the IBS-C respondents were identified as having IBS-C based on either patient report of a formal diagnosis by an HCP or a screening questionnaire using Rome IV criteria. Lastly, the HCPs, though screened for experience in the management of patients with IBS-C, may have self-selected based on interest in study participation.

\section{CONCLUSION}

The BURDEN IBS-C study is the second largescale patient and HCP questionnaire-the first being BURDEN-CIC [5] - to highlight the frustration and high degree of dissatisfaction with OTC laxatives and prescription therapies in the management of IBS-C. Despite this agreement on dissatisfaction, HCPs did not perceive patients as accepting of their disorder, while patients expressed a high level of physical and psychosocial acceptance of their IBS-C, suggesting an opportunity for HCPs to bridge this divide in the management and experience of their patients with IBS-C. In addition, this study identified several barriers to improving outcomes for IBS-C sufferers, including the finding that many respondents had not consulted a medical professional and/or were not currently using any treatments for their IBS-C symptoms. Symptomatic individuals who do not seek professional medical advice may be at a disadvantage for achieving symptom relief. Greater awareness of functional constipation as a treatable medical issue is needed to promote HCP visits and initiate a course of treatment. For patients diagnosed with IBS-C, there was a low percentage of prescription drug use, which was not evaluated in depth in this study, though anticipated factors such as inadequate efficacy, side effects, attitude toward symptoms, and insurance coverage may play a role in the decision to step up to prescription treatment. Lastly, there were observed differences between types of HCPs (i.e., GEs, PCPs, NPs, and PAs) in regard to challenges to IBS-C management and outcome expectations, suggesting that the relationships between patients and their HCP may differ depending on the HCP type and that a more standardized approach to treatment may be warranted. Indeed, many of the large variety of treatment recommendations and approaches are based on weak clinical study evidence as described in current treatment guidelines $[2,4]$. Ongoing and improved dialogue is needed between HCPs and their IBS-C patients, as well as additional treatment options, to ensure appropriate treatment expectations and outcomes are achieved.

\section{ACKNOWLEDGEMENTS}

We thank the participants of the study.

Funding. Synergy Pharmaceuticals Inc. provided funding for this study, manuscript support, and article processing charges, including open access fees. All authors had full access to all of the data in this study and take complete responsibility for the integrity of the data and accuracy of the data analysis.

Medical Writing and Editorial Assistance. Writing and editorial support were provided by Nicole Coolbaugh of The Medicine Group (New Hope, PA, USA), which was funded by Synergy Pharmaceuticals Inc.

Authorship. All named authors meet the International Committee of Medical Journal Editors (ICMJE) criteria for authorship for this manuscript, take responsibility for the integrity of the work as a whole, and have given final approval for the version to be published.

Disclosures. Eamonn M.M. Quigley has participated in advisory boards and has served as a consultant for Synergy Pharmaceuticals Inc. Lucinda A. Harris has participated in advisory boards and has served as a consultant for Synergy Pharmaceuticals Inc. Robert A. Crozier is an employee and holder of stock options in Synergy Pharmaceuticals Inc. Michele Kissous-Hunt has served on speakers bureaus for Synergy Pharmaceuticals Inc. John Horn has nothing to disclose. 
Compliance with Ethics Guidelines. The patient protocol and associated materials were reviewed and approved by the Western Institutional Review Board. GfK conducted both the patient and HCP questionnaires in accordance with globally accepted standards of good clinical practice (as defined in the International Conference on Harmonisation E6 Guideline for Good Clinical Practice), in accordance with the Health Insurance Portability and Accountability Act, and in keeping with local regulations. Informed consent was obtained from all respondents before being included in the study.

Data Availability. The datasets during and/ or analyzed during the current study are available from the corresponding author on reasonable request.

Open Access. This article is distributed under the terms of the Creative Commons Attribution-NonCommercial 4.0 International License (http://creativecommons.org/licenses/ by-nc/4.0/), which permits any noncommercial use, distribution, and reproduction in any medium, provided you give appropriate credit to the original author(s) and the source, provide a link to the Creative Commons license, and indicate if changes were made.

\section{REFERENCES}

1. Hungin AP, Chang L, Locke GR, Dennis EH, Barghout V. Irritable bowel syndrome in the United States: prevalence, symptom patterns and impact. Aliment Pharmacol Ther. 2005;21:1365-75.

2. Lacy BE, Mearin F, Chang L, et al. Bowel disorders. Gastroenterology. 2016;150(1393-407):e5.

3. Trinkley KE, Sill BE, Porter K, Nahata MC. Prescribing patterns for outpatient treatment of constipation, irritable bowel syndrome-related constipation, and opioid-induced constipation: a retrospective cross-sectional study. J Manag Care Spec Pharm. 2015;21:1077-87.

4. Ford AC, Moayyedi P, Lacy BE, et al. American College of Gastroenterology monograph on the management of irritable bowel syndrome and chronic idiopathic constipation. Am J Gastroenterol. 2014;109(Suppl 1):S2-26 (quiz S7).

5. Harris LA, Horn J, Kissous-Hunt M, Magnus L, Quigley EMM. The better understanding and recognition of the disconnects, experiences, and needs of patients with chronic idiopathic constipation (BURDEN-CIC) study: results of an online questionnaire. Adv Ther. 2017;34:2661-73.

6. Knowledge Panel ${ }^{\circledR}$ Recruitment and sample survey methodologies. http://www.gfk.com/fileadmin/ user_upload/dyna_content/US/documents/Knowled gePanel_Methodology.pdf. Accessed 20 Mar 2018.

7. Heidelbaugh JJ, Stelwagon M, Miller SA, Shea EP, Chey WD. The spectrum of constipation-predominant irritable bowel syndrome and chronic idiopathic constipation: US survey assessing symptoms, care seeking, and disease burden. Am J Gastroenterol. 2015;110:580-7.

8. DiBonaventura M, Sun SX, Bolge SC, Wagner JS, Mody R. Health-related quality of life, work productivity and health care resource use associated with constipation predominant irritable bowel syndrome. Curr Med Res Opin. 2011;27:2213-22.

9. American Gastroenterological Association. American Gastroenterological Association (AGA). IBS in America-survey summary findings 2015. http:// www.multivu.com/players/English/7634451-aga-ibsin-america-survey/docs/survey-findings-pdf-6354731 72.pdf. Accessed 20 Mar 2018.

10. Saito YA, Schoenfeld P, Locke GR 3rd. The epidemiology of irritable bowel syndrome in North America: a systematic review. Am J Gastroenterol. 2002;97:1910-5.

11. Drossman DA, Chang L, Bellamy N, et al. Severity in irritable bowel syndrome: a Rome Foundation Working Team report. Am J Gastroenterol. 2011;106:1749-59 (quiz 60).

12. Lee OY, Mayer EA, Schmulson M, Chang L, Naliboff B. Gender-related differences in IBS symptoms. Am J Gastroenterol. 2001;96:2184-93.

13. Coffin B, Dapoigny M, Cloarec D, Comet D, Dyard F. Relationship between severity of symptoms and quality of life in 858 patients with irritable bowel syndrome. Gastroenterol Clin Biol. 2004;28:11-5.

14. Bjorkman I, Dellenborg L, Ringstrom G, Simren M, Jakobsson Ung E. The gendered impact of irritable bowel syndrome: a qualitative study of patients' experiences. J Adv Nurs. 2014;70:1334-43.

15. Spiegel BM, Gralnek IM, Bolus R, et al. Clinical determinants of health-related quality of life in 
patients with irritable bowel syndrome. Arch Intern Med. 2004;164:1773-80.

16. Quigley BM, Sova CC, Brenner DM, et al. (Can't get no) Patient satisfaction: the predictive power of demographic, GI, and psychological factors in IBS patients. J Clin Gastroenterol. 2017;152(Suppl 1):S173.
17. Neri L, Iovino P. Bloating is associated with worse quality of life, treatment satisfaction, and treatment responsiveness among patients with constipationpredominant irritable bowel syndrome and functional constipation. 2016;28:581-91. 